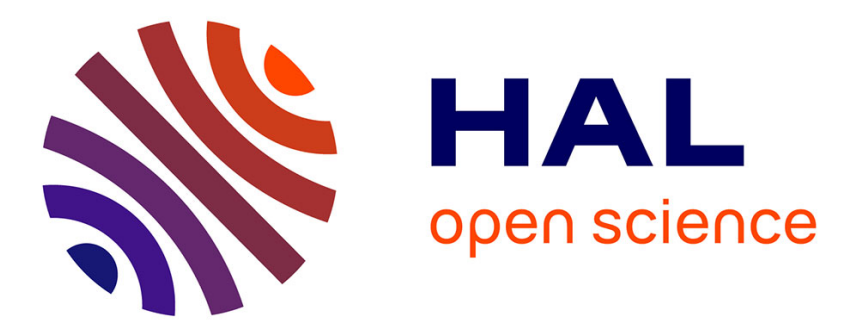

\title{
Sound fields separation and reconstruction of irregularly shaped sources
}

Nicolas Totaro, Dorian Vigoureux, Q. Leclere, J Lagneaux, Jean-Louis

Guyader

\section{- To cite this version:}

Nicolas Totaro, Dorian Vigoureux, Q. Leclere, J Lagneaux, Jean-Louis Guyader. Sound fields separation and reconstruction of irregularly shaped sources. Journal of Sound and Vibration, 2015, 336, pp.62-81. 10.1016/j.jsv.2014.09.040 . hal-01275283

\section{HAL Id: hal-01275283 \\ https://hal.science/hal-01275283}

Submitted on 19 Feb 2016

HAL is a multi-disciplinary open access archive for the deposit and dissemination of scientific research documents, whether they are published or not. The documents may come from teaching and research institutions in France or abroad, or from public or private research centers.
L'archive ouverte pluridisciplinaire HAL, est destinée au dépôt et à la diffusion de documents scientifiques de niveau recherche, publiés ou non, émanant des établissements d'enseignement et de recherche français ou étrangers, des laboratoires publics ou privés. 


\title{
Sound fields separation and reconstruction of irregularly shaped sources
}

\author{
N. Totaro ${ }^{1}$, D. Vigoureux, Q. Leclère, J. Lagneaux and J.L Guyader \\ Laboratoire Vibrations Acoustique - INSA-Lyon - Bâtiment St Exupery \\ 20, avenue Albert Einstein - F69621 Villeurbanne Cedex - France
}

\begin{abstract}
Nowadays, the need of source identification methods is still growing and application cases are more and more complex. As a consequence, it is necessary to develop methods allowing to reconstruct sound fields on irregularly shaped sources in reverberant or confined acoustic environment.

The inverse Patch Transfer Functions (iPTF) method is suitable to achieve these objectives. Indeed, as the iPTF method is based on Green's identity and double measurements of pressure and particle velocity on a surface surrounding the source, it is independent of the acoustic environment. In addition, the finite element solver used to compute the patch transfer functions permits to handle sources with 3D irregular shapes.

In the present article, two experimental applications on a flat plate and an oil pan have been carried out to show the performances of the method on real applications. As for all ill-posed problem, it is shown that the crucial point of this method is the choice of the parameter of the Tikhonov regularization, one of the most widely used in the literature. The classical L-curve strategy sometimes fails to choose the best solution. This issue is clearly explained and an adapted strategy combining L-curve and acoustic power conservation is proposed. The efficiency of this strategy is demonstrated on both applications and compared to results obtained with Generalized Cross Validation (GCV) technique.
\end{abstract}

Keywords: source identification, underdetermined inverse problem, source separation, Tikhonov regularization, Generalized Cross Validation

1. Corresponding author. Tel. : +33172438082. E-mail address : nicolas.totaro@insalyon.fr

Preprint submitted to Journal of Sound and Vibration

23 février 2015 


\section{Introduction}

In acoustics, inverse methods cover a wide field of applications. Nevertheless, they all rely on the same concept : identifying the inputs of a linear system by observing the outputs. If the observed outputs are in most cases the pressure and/or particle velocity fields radiated by the source, the objectives of the identification processes can be very different. Indeed, the term identification methods encompasses goals as different as source localization in wide acoustic spaces, source field reconstruction on academic or complex geometries, noise source identification in enclosed sound fields, source separation or source ranking. A unique method to treat all these applications does not exist and several specialized methods have been developed since decades [1].

The Near-field Acoustic Holography $(\mathrm{NAH})$ is one of the most widely used method. Firstly introduced by Williams et al [2] and Maynard et al [3], this Fourier approach reconstructs the sound sources using a spatial 2D Fourier transform of the Helmholtz equation. Initially, NAH was developped to be used with a planar array of microphones but has been extended to cylindrical [4] or spherical geometries [5]. Many alternative methods, all based on NAH, can be found in the literature. One can cite, for example, the works done by Chardon et al [6] who use the property of sparsity of signals in NAH. NAH can also be coupled to force identification method [7] to provide a complete pipeline from radiated noise to structural excitation.

A second category of identification methods is based on equivalent sources. In this type of methods, the source (eventually irregularly shaped) are replaced by a set of monopoles and dipoles $[8,10]$ and the unknowns are the strengths of equivalent sources.

A third category is based on solving the Helmholtz-Kirchhoff equation by Boundary Element Method (BEM) [11, 12, 13] or Finite Element Method (FEM) [14, 15]. The identification method used in the present article belongs to the latter. The inverse Patch Transfer Functions (iPTF) method, firstly introduced by Aucejo [14] and Vigoureux [16] is aimed to reconstruct the velocity field of a radiating structure using acoustic measurements. The basic idea of the iPTF method is based on the use of the Green identity on a finite acoustic volume. It can be considered as a data completion method following the definition given by Langrenne and Garcia [17] : "the acoustic 
gradients and pressures are known on a surface surrounding the source, but are unknown on its structure".

In addition, iPTF can also be considered as a separation method [18, 19] as it permits to identify a source in small spaces or in non-anechoic acoustic environment.

The iPTF method has been applied with success on baffled pistons with the presence of external correlated sources [20], on L-shaped plate [21] and on irregularly shaped structures such as a guitar [22], a precatalyst of a diesel engine [23] or a reduction gearbox [24].

However, as all inverse methods [25, 26], the iPTF suffers from problems associated with the inversion of an ill-posed problem. The objective of the present article is to understand the physical origin of that ill-conditioning by deeply analysing the Tikhonov regularization process, one of the most widely used in the literature. As usual, the choice of the regularization parameter is crucial. It is demonstrated that, at some frequencies, the well-known L-curve strategy fails. This is due to the fact that L-curve exhibits more than one corner. The presence of these multiple corners is explained and a modified Lcurve strategy, called weighted curvature strategy, is proposed and compared to results obtained with Generalized Cross Validation (GCV) technique. Two experiments are used to illustrate the regularization process and to evaluate capabilities of the iPTF method : a flat plate and an oil pan.

\section{Inverse Patch Transfer Function (iPTF) method}

In the example of Fig. 1, a vibrating irregularly shaped surface radiates in any acoustic environment. The objective of the iPTF method is to separate the sound field radiated by the source and the sound field coming from the acoustic environment (disturbing sources, reflections, etc.). This is achieved by defining a finite acoustic volume $\Omega$ surrounding this vibrating surface as presented in Fig. 1. This virtual volume is delimited by surfaces $\Sigma$ (surface of the vibrating object), $\Gamma$ (an arbitrarily chosen virtual surface surrounding the object) and $\Lambda$ (a physically rigid surface). In that case, the Green's identity writes

$$
\begin{gathered}
\iiint_{\Omega}(\Psi \Delta \Phi-\Phi \Delta \Psi) d \Omega=\iint_{\Sigma}\left(\Phi \frac{\partial \Psi}{\partial n}-\Psi \frac{\partial \Phi}{\partial n}\right) d \Sigma+ \\
\iint_{\Gamma}\left(\Phi \frac{\partial \Psi}{\partial n}-\Psi \frac{\partial \Phi}{\partial n}\right) d \Gamma+\iint_{\Lambda}\left(\Phi \frac{\partial \Psi}{\partial n}-\Psi \frac{\partial \Phi}{\partial n}\right) d \Lambda .
\end{gathered}
$$


In Eq. (1), the functions $\Phi$ and $\Psi$ can be arbitrarily chosen (provided that they are defined and twice differentiable on the domain). In the following, the function $\Phi$ will be the acoustic pressure $p$ defined by the Helmholtz equation (considering there is no source in the volume)

$$
\Delta p(M)+k^{2} p(M)=0, \forall M \in \Omega,
$$

and boundary conditions on surfaces $\Sigma, \Gamma$ and $\Lambda$ are given by :

$$
\begin{gathered}
\frac{\partial p(N)}{\partial \vec{n}}=0, \forall N \in \Lambda, \\
\frac{\partial p(Q)}{\partial \vec{n}}=-\mathrm{i} \omega \rho_{0} V(Q), \forall Q \in \Gamma
\end{gathered}
$$

and

$$
\frac{\partial p\left(Q^{\prime}\right)}{\partial \vec{n}}=-i \omega \rho_{0} V\left(Q^{\prime}\right), \forall Q^{\prime} \in \Sigma
$$

where $V(Q)$ is the normal velocity at each point $Q$ of the surface and $\vec{n}$ is the outer normal of the volume $\Omega, \rho_{0}$ is the density of air, $\omega$ is the angular frequency and $k$ the acoustic wavenumber.

The function $\Psi$ of the Green's identity is chosen to be the eigenmodes $\phi_{n}$ of the virtual acoustic volume respecting Helmholtz equation :

$$
\Delta \phi_{n}(M)+k_{n}^{2} \phi_{n}(M)=0, \forall M \in \Omega,
$$

where $k_{n}$ is the natural wavenumber of mode $n$. The boundary conditions on surfaces $\Sigma, \Gamma$ and $\Lambda$ are given by :

$$
\begin{gathered}
\frac{\partial \phi_{n}(N)}{\partial n}=0, \forall N \in \Lambda, \\
\frac{\partial \phi_{n}(Q)}{\partial n}=0, \forall Q \in \Gamma
\end{gathered}
$$

and

$$
\frac{\partial \phi_{n}\left(Q^{\prime}\right)}{\partial n}=0, \forall Q^{\prime} \in \Sigma
$$

These chosen eigenmodes satisfy the homogeneous Neumann's problem. This is an arbitrary choice and does not correspond to the boundary conditions of the real problem. The eigenmodes are only used here as a mathematical basis of functions to solve the homogeneous Helmholtz equation. This is why the 
acoustic volume delimited by surfaces $\Gamma, \Sigma$ and $\Lambda$ is called virtual volume. It is not a closed volume but just a part of the acoustic environment of the source.

The velocity of the virtual surface will represent the presence of the acoustic environment outside the virtual volume even if it is reverberant, geometrically complex or even if it contains disturbing sources (sources outside the volume of interest).

Solving the Helmholtz Eq. (2) using decomposition on modes $\phi_{n}(M)$, one can express the pressure at any point of the volume $\Omega$ as a function of normal velocities of surfaces $\Gamma$ and $\Sigma$

$$
\begin{aligned}
p(M)= & \mathrm{i} \omega \rho_{0} \sum_{n=1}^{\infty} \frac{\phi_{n}(M)}{\chi_{n}\left(k^{2}-k_{n}^{2}\right)} \iint_{\Gamma} \phi_{n}(Q) V(Q) d Q+ \\
& +\mathrm{i} \omega \rho_{0} \sum_{n=1}^{\infty} \frac{\phi_{n}(M)}{\chi_{n}\left(k^{2}-k_{n}^{2}\right)} \iint_{\Sigma} \phi_{n}\left(Q^{\prime}\right) V\left(Q^{\prime}\right) d Q^{\prime},
\end{aligned}
$$

where $\chi_{n}$ is the norm of mode $n$.

Due to the boundary conditions given by Eqs. (3) and (7), the presence of the physically rigid surface $\Lambda$ does not appear in the expression of the pressure. Indeed, the presence of the rigid wall is already taken into account in the modal basis of modes $\phi_{n}(M)$. This can be an additional advantage of using eigenmodes of the virtual volume : this volume can be inhomogeneous and reflections on some rigid structures (a heavy frame for example) placed inside the virtual volume are taken into account by eigenmodes.

Dividing the surfaces $\Gamma$ and $\Sigma$ into elementary surfaces - called patches $\Gamma_{i}$ (or $\Gamma_{j}$ ) and $\Sigma_{k}$ (or $\Sigma_{l}$ ) - the integrals are replaced by sums in Eq. (10). The averaged pressure on one patch of the surface $\Gamma$ can be expressed as a function of velocities of patches $\Gamma_{j}$ and $\Sigma_{k}$ (of areas $S_{j}$ and $S_{k}$ )

$$
\langle p\rangle_{i}=\mathrm{i} \omega \rho_{0} \sum_{j=1}^{N_{j}}\langle V\rangle_{j} \sum_{n=1}^{\infty} \frac{S_{j}\left\langle\phi_{n}\right\rangle_{i}\left\langle\phi_{n}\right\rangle_{j}}{\chi_{n}\left(k^{2}-k_{n}^{2}\right)}+\mathrm{i} \omega \rho_{0} \sum_{k=1}^{N_{k}}\langle V\rangle_{k} \sum_{n=1}^{\infty} \frac{S_{k}\left\langle\phi_{n}\right\rangle_{i}\left\langle\phi_{n}\right\rangle_{k}}{\chi_{n}\left(k^{2}-k_{n}^{2}\right)}
$$

where $\langle\bullet\rangle_{j}=\frac{1}{S_{j}} \int_{S_{j}} \bullet d S$ represents the space average on a patch $j$.

The pressures on patches $j$ of the surface $\Gamma$ can be written in a matrix form

$$
\mathbf{p}_{i}=\mathbf{Z}_{i j} \mathbf{V}_{j}+\mathbf{Z}_{i k} \mathbf{V}_{k}
$$


where $\mathbf{Z}_{i j}$ (resp. $\mathbf{Z}_{i k}$ ) is an impedance matrix where each term is defined by the ratio between the mean pressure on patch $i$ and the mean normal velocity on patch $j$ (resp. $k$ )

$$
Z_{i j}=\frac{\langle p\rangle_{i}}{\langle V\rangle_{j}} .
$$

As a consequence by inverting Eq. (12) one can express the source velocities $\mathbf{V}_{k}$ (on patches $\Sigma_{k}$ ) as a function of pressures $\mathbf{p}_{i}$ and velocities $\mathbf{V}_{j}$ measured on the virtual surface $\Gamma$.

Finally, the source velocities are obtained solving

$$
\mathbf{V}_{k}=\mathbf{Z}_{i k}^{-1}\left(\mathbf{p}_{i}-\mathbf{Z}_{i j} \mathbf{V}_{j}\right)
$$

Even if the source velocity is the primary unknown, it is possible with no additional inversion of matrix, to compute the boundary pressure $\mathbf{p}_{k}$ on source surface. Indeed, the pressure on the surface of the source is due to the contribution of the velocity of the source $\mathbf{V}_{l}$ (identified during the previous step) and to the contribution of the particle velocity $\mathbf{V}_{j}$ of the virtual surface (already measured) :

$$
\mathbf{p}_{k}=\mathbf{Z}_{k j} \mathbf{V}_{j}+\mathbf{Z}_{k l} \mathbf{V}_{l} \text {. }
$$

The normal intensity is simply deduced from :

$$
\mathbf{I}_{k}=\frac{1}{2} \Re\left(\mathbf{p}_{k} \circ \mathbf{V}_{k}^{*}\right),
$$

where $\Re(\bullet)$ and $\bullet *$ denote the real part and the complex conjugate of a complex number. $\circ$ represents the Hadamard's product.

Thus, the acoustic source is completely characterized by the identification process. At the end, global indicators like active and reactive acoustic power and radiation efficiency of the source can also be produced with no effort (neither numerically nor experimentally).

\section{Classical Tikhonov's regularization strategies}

\subsection{Ill-posed and underdetermined problems}

The primary unknown of the problem is the source velocity on surface $\Gamma$. To obtain this source velocity, the inversion of an ill-conditioned matrix is needed. Eq. (14) has the classical form $\mathbf{A x}=\mathbf{b}$

$$
\mathbf{Z}_{i k} \mathbf{V}_{k}=\tilde{\mathbf{p}}_{i}
$$


where $\tilde{\mathbf{p}}_{i}=\mathbf{p}_{i}-\mathbf{Z}_{i j} \mathbf{V}_{j}$. The impedance matrix $\mathbf{Z}_{i k}$ is computed using modes of the virtual volume with rigid walls extracted by standard finite element methods. This matrix represents the model used to represent the system. The vector $\tilde{\mathbf{p}}_{i}$ is measured with pU probes [27] on the virtual surface $\Gamma$. This vector represents the output of the system. This vector is obviously affected by measurement noise. The objective here is to deduce the input of the system (the source velocities) knowing the model and measuring the output of the system.

This problem is a classical ill-posed problem as defined by Hansen [25]. If $N_{k}$ (the number of patches on the source surface) is higher than $N_{j}$ (the number of patches on the virtual surface), then the problem is underdetermined and many regularization techniques can be used. As this ill-posed problem is classic in acoustic holography, these techniques have been extensively studied in the literature. The most known method is the Tikhonov regularization but alternative approaches like the Truncated Singular Values Decomposition [25], the Bayesian regularization [28, 29], the sparse regularization [6] or the $l_{1}$-norm minimization [30] also exist.

The Tikhonov regularization is based on the minimization of a functional $J$ defined as the sum of the residual norm and a penalty term proportional to the norm of the regularized solution

$$
J=\left\|\mathbf{Z}_{i k} \mathbf{V}_{k}-\tilde{\mathbf{p}}_{i}\right\|_{2}+\beta\left\|\mathbf{L} \mathbf{V}_{k}\right\|_{2}
$$

where $\|\bullet\|_{2}$ is the $l_{2}$-norm, $\beta$ is the regularization parameter and $\mathbf{L}$ is a non-negative definite matrix often assumed to be the identity matrix. The crucial point of the Tikhonov regularization is the choice of this regularization parameter. A small $\beta$ value (less filtering) gives more importance to the minimization of the residual norm so that the solution fits well with measurements. However, the norm of this solution is generally high in that case. Conversely, a high $\beta$ value (more filtering) gives more importance to the minimization of the norm of the solution but the solution deviates from the measurements and the residual norm increases. The good choice of $\beta$ is a balance between these two opposite behaviors.

Using the Singular Values Decomposition (SVD) of the matrix $\mathbf{Z}_{i k}$, the regularized solution $\mathbf{V}_{k}^{\mathrm{R}}$ that minimizes the functional $J$ is given by

$$
\mathbf{V}_{k}^{\mathrm{R}}=\sum_{m=1}^{N_{V S}} \frac{\sigma_{m}^{2}}{\sigma_{m}^{2}+\beta} \frac{\mathbf{u}_{m}^{\mathrm{H}} \tilde{\mathbf{p}}_{i}}{\sigma_{m}} \mathbf{v}_{m}
$$


where $\sigma_{m}$ are singular values in a decreasing order, $\mathbf{u}_{m}$ and $\mathbf{v}_{m}$ are the left and right singular vectors of matrix $\mathbf{Z}_{i k}$ and $N_{V S}$ is the number of singular values (equal to $N_{j}$ the number of patches on the virtual surface).

In fact, the Tikhonov regularization acts like a low pass filter on singular values given by filter factor

$$
f_{m}=\frac{\sigma_{m}^{2}}{\sigma_{m}^{2}+\beta} .
$$

For TSVD, the filter factor is step-like (1 or 0$)$. The Tikhonov filter factor is smoother and continuously weights the singular values as a function of $\beta$ parameter. The choice of $\beta$ is, in turn, the choice of a threshold on the acceptable singular values [31].

The search of the optimal regularization parameter $\beta$ for Tikhonov regularization has produced a lot of interesting studies in the framework of acoustical inverse problems. For instance, the performances of the L-curve and the Generalized Cross Validation methods (two known regularization parameterdetermination methods) have been studied by Williams [26], Leclère [32] and Kim and Nelson [33].

\subsection{Curvature of the L-curve}

The L-curve is a widely used technique to select the optimal regularization parameter $\beta$. The L-curve plots the evolution of the two terms of Eq. (18) : the norm of the regularized solution as a function of the residual norm. The compromise between both terms is located at the corner of the curve which is generally L-shaped.

To easily find the $\beta$ value corresponding to the corner of the L-curve, the curvature function is generally used [31, 32, 34, 35]. A corner of the L-curve corresponds to a maximum value of the curvature function.

\subsection{Generalized Cross Validation}

The Generalized Cross Validation [31, 32, 34, 35] is an alternative to the L-curve to determine the optimal parameter $\beta$. This technique is based on the minimization of the GCV function given by

$$
G C V(\beta)=\frac{\left\|\mathbf{I}-\mathbf{B}(\beta) \tilde{\mathbf{p}}_{i}\right\|_{2}^{2}}{[\operatorname{Tr}(\mathbf{I}-\mathbf{B}(\beta))]^{2}}
$$

where the influence matrix is $\mathbf{B}(\beta)=\mathbf{Z}_{i k}\left(\mathbf{Z}_{i k}^{\mathrm{H}} \mathbf{Z}_{i k}+\beta \mathbf{I}\right)^{-1} \mathbf{Z}_{i k}^{\mathrm{H}}$ 


\section{2D underdetermined problem and regularization process}

\subsection{System under study}

To evaluate the ability of iPTF method in the reconstruction of source field, two experimental validations have been set up : one 2D application on an academic structure and one 3D application on an industrial structure. The first system under study consists in an aluminium rectangular plate glued to a wood frame as can be seen in Fig. 2. The free surface of the plate is $60 \mathrm{~cm}$ long and $30 \mathrm{~cm}$ large. The plate is excited by an electro-dynamic shaker fed with a white noise between 100 and $1000 \mathrm{~Hz}$ and radiates in a quiet room. The measurements have thus not been done in free field conditions. However, the iPTF method has been developed to isolate the object from its acoustic environment as previously explained. The iPTF method is considered as a field separation technique thanks to the use of the double information pressure/particle velocity on a surface surrounding the source.

A pU probe has been used to measure the acoustic pressure and the particle velocity on :

- a $17 \times 10$ grid mesh (see Fig. 3(a)) to have reference fields of source velocity, boundary pressure and normal intensity. These reference measurements will be used to evaluate the iPTF capabilities.

- a virtual surface (a $5 \mathrm{~cm}$ high rectangular box) presented in Fig. 3(b) divided into 535 patches. The acoustic pressure and the particule velocity have been measured at center of each patch. These measurements are used to compute vector $\tilde{\mathbf{p}}_{i}$ in Eq. (17).

As the measurements on the grid meshes are sequential, a phase reference (an accelerometer) has been glued on the surface of the plate.

The iPTF method is applied here to reconstruct the source fields on a $45 \times 25$ identification grid mesh, see Fig. 3(c). The problem is then highly underdetermined as there are 1125 patches of identification (on the surface of the plate) and only 535 patches of measurements (on the virtual surface).

To compute the matrix $\mathbf{Z}_{i k}$ in Eq. (17) a finite element model, shown in Fig. $3(\mathrm{~d})$, has been used to extract 1883 modes of the virtual volume up to $11 \mathrm{kHz}$ (respecting a $\lambda / 6$ criterion for the $\mathrm{FE}$ mesh size).

The condition number of the matrix $\mathbf{Z}_{i k}$ is plotted in Fig. 4 as a function of frequency and some additional information about numerical computations are given in Tab. 1. It is interesting to notice that the condition number exhibits peaks in the whole frequency range. As presented in Fig. 4, these peaks 


\begin{tabular}{|c|c|}
\hline length $\times$ width $\times$ height $(\mathrm{m})$ & $0.6 \times 0.3 \times 0.05$ \\
\hline Number of measurement patches $N_{j}$ & 535 \\
\hline Number of identification patches $N_{k}$ & 1125 \\
\hline Maximum frequency of the modal basis & $11 \mathrm{kHz}$ \\
\hline Number of modes & 1883 \\
\hline Number of DoF & 93632 \\
\hline Rank of the matrix $\mathbf{Z}_{i k}$ & 535 \\
\hline
\end{tabular}

TABLE 1: Information on the virtual volume used to compute impedance matrix $\mathbf{Z}_{i k}$ in the case of the rectangular plate.

exactly correspond to the eigenfrequencies of the virtual volume (plotted in dashed lines). This particularity is discussed in the following.

\subsection{Identification results}

In Fig. 5, the identified mean square velocity, mean square pressure and acoustic power are compared to the reference measurements. The measures on the virtual surface are also plotted to show how different are the radiated and the reference fields.

Both the CURV (maximum of the curvature of the L-curve) and the GCV strategies have been applied. Both regularization parameter-determination methods compare well in the whole frequency range except at two particular frequencies. Indeed, at $250 \mathrm{~Hz}$ and $880 \mathrm{~Hz}$, the results obtained using CURVstrategy seem to deviate from reference measurements as shown in Fig. 5(b). To understand why the CURV-strategy fails to identify the good solution for some frequencies, an insight into regularization process has been carried out at two frequencies : at $137.5 \mathrm{~Hz}$ (where both CURV-strategy and GCV-strategy give satisfactory results) and at $250 \mathrm{~Hz}$ (where CURV-strategy fails).

Fig. 6 shows L-curve, curvature function, GCV function and power ratio $\Pi(\beta)$ as a function of regularization parameter $\beta$. The power ratio is the ratio between the acoustic power $\Pi_{\Sigma}^{\mathrm{id}}(\beta)$ identified on the surface $\Sigma$ of the vibrating object for each $\beta$ value and the acoustic power $\Pi_{\Gamma}^{\text {meas }}$ measured on the virtual surface $\Gamma$ as defined in Eq. (22).

$$
\Pi(\beta)=\frac{\Pi_{\Sigma}^{\mathrm{id}}(\beta)}{\Pi_{\Gamma}^{\mathrm{meas}}}
$$

The power ratio is obviously independent of the reference acoustic power and is obtained using quantities needed (pressure and particule velocity on 
the virtual surface) or produced (pressure and velocity on the identification surface) by iPTF method. If the power conservation is achieved, the power ratio tends to unity.

At $137.5 \mathrm{~Hz}$, both CURV and GCV strategies produce good results. At this frequency, the L-curve is indeed L-shaped. The optimal $\beta$ values (represented by a cross for CURV-strategy and by a square for GCV-strategy) correspond to the (unique) corner of the L-curve even if they are a little bit different $\left(\beta^{\mathrm{Curv}}=712.2\right.$ and $\left.\beta^{\mathrm{GCV}}=2270\right)$. As a consequence, $\beta^{\mathrm{GCV}}$ does not correspond exactly to the maximum of curvature of the L-curve and $\beta^{\text {Curv }}$ does not correspond exactly to the minimum of the GCV function. It can be noticed that both strategies imply a power ratio close to 1 .

Fig. 7 demonstrates and confirms that both strategies produce satisfactory identified fields compared to reference. Precise details can be observed with both strategies.

At $250 \mathrm{~Hz}$, the Curv-strategy fails as can be seen in Fig. 5(b). This is mainly due to the non-uniqueness of the corner of the L-curve. Indeed, in that case, Fig. 6 shows that the L-curve is rather a $\mathrm{W}$-shaped curve and exhibits two corners (multiple corners of L-curve have been also observed by Pereira $[9,10]$ using Equivalent Source Method). These two corners result in two curvature maxima. As can be seen in Fig. 6(d), the CuRV-strategy chooses the highest curvature value that corresponds here to the second peak where $\beta^{\text {Curv }}=1.416 \times 10^{8}$. For the first peak of curvature function (which is not automatically chosen by CuRV-strategy), the $\beta$ value is 179.5 . At this frequency, the $\beta^{\text {Curv }}$ value is completely different from the one defined by GCV-strategy $\left(\beta^{G C V}=600\right)$ which is closer to the value of the first peak of curvature function. It is interesting to notice that it is difficult to distinguish two clearly different possibilities for $\beta^{\mathrm{GCV}}$ as with curvature function. One can at most distinguish inflection of the GCV curve around $\beta^{\text {Curv }}=1.416 \times 10^{8}$. GCVstrategy seems to be less affected than CuRv-strategy by the high condition number of the matrix to be inverted at this frequency.

An important remark raises from the analysis of the power ratio in Fig. 6(h). For $\beta^{G C V}$, the power ratio is still close to 1 but for $\beta^{\text {Curv }}$, the solution identified has a power ratio less than 0.3 indicating that the power conservation is not achieved in that case. This is due to an excessive regularization (Eq. $18)$.

On the contrary, the power conservation is achieved for the first peak of the curvature function. Analysing maps obtained with this $\beta$ value (noted WCURV in Fig. 6), one can see on Fig. 8 that this solution produces more 
reliable results.

\subsection{The WCURV-strategy}

To overcome this issue, it is possible to weight the curvature function by the power ratio function to filter the solution for which the power conservation is not achieved. The WCURV-strategy (weighted curvature strategy) only consists in finding the maximum of function $\operatorname{wCurv}(\beta)$ defined by

$$
{ }_{w} \operatorname{Curv}(\beta)=\left\{\begin{array}{cl}
\operatorname{Curv}(\beta) \Pi(\beta) & \text { if } \Pi(\beta)>0 \\
0 & \text { if } \Pi(\beta)<0
\end{array}\right.
$$

where $\operatorname{Curv}(\beta)$ is the curvature function and $\Pi(\beta)$ is the power ratio function given by Eq. (22). The WCURV function is plotted in Fig. 6(d) (in dashed line). The optimal $\beta^{\text {wCurv }}$ values obtained with wCURV-strategy are represented by triangles in Fig. 6 .

Fig. 9 presents the effect of the weighting on the curvature function as a function of frequency and $\beta$ value. Fig. 9(a) shows that wrong solutions may appear at frequencies corresponding to the eigenfrequencies of the virtual volume with rigid walls (the associate problem) (vertical dashed lines). The weighting filters out second peak of curvature that produces wrong solutions. WCURV-strategy takes advantage of information available during the iPTF identification process.

The iPTF method can be experimentally constraining because it needs pressure and particle velocity on a virtual surface surrounding the source, but it provides a complete characterization of the source from the velocity field to the acoustic power, the active and reactive intensity and also the radiation efficiency as presented in Fig. 10. The very low radiation efficiency of the plate in this frequency band is validated by the Crocker and Price's model [36].

\subsection{Interpretation of wrong solutions of CURV-strategy}

WCURV-strategy permits to overcome wrong choices of CURV-strategy by promoting solutions that respects the power conservation. However, this does not explain why such solutions appear with CURV-strategy.

Analysing the filter factors (Eq. (20)) obtained with $\beta$ defined by the first and the second peak of curvature observed at $250 \mathrm{~Hz}$, one can see that CURVstrategy $\left(\beta^{\text {Curv }}=1.416 \times 10^{8}\right)$ strongly filter singular values. Indeed, with 
such a regularization parameter, only two singular values have a filter factor higher than 0.1 which means that almost all the singular values are filtered as shown in Fig. 11. On the contrary, with $\beta$ value obtained with wCURVstrategy $\left(\beta^{\text {wCurv }}=179.5\right)$, a lot of singular values participate (187 singular values have a filter factor higher than 0.1).

As a consequence, the wrong solution of CURV-strategy is highly dominated by the first singular value of the matrix. Comparing this wrong solution to the projection of the second mode (eigenfrequency $=250 \mathrm{~Hz}$ ) of the virtual volume on the surface of the plate, it is easy to conclude that the first singular value of the matrix is highly dominated by the eigenmode of the virtual cavity, see Fig. 12. The same conclusion holds at $880 \mathrm{~Hz}$. The identified velocity field highly correlates to the pressure mode shape of the eighth modes (eigenfrequency $=873.5 \mathrm{~Hz}$ ).

In the case of single layer pressure-velocity (as it is the case for iPTF), Fernandez-Grande et al. [18] propose to use a weighted least squares solution. This is necessary beacause the solution vector is, in that case, composed by pressures and velocities

$$
\left[\begin{array}{l}
\mathbf{p}_{h} \\
\mathbf{u}_{h}
\end{array}\right]
$$

with completely different amplitudes. The weighting is supposed to reduce the problem, so that the energies of the pressure and velocity observations are similar. This is not the case in iPTF method. Indeed, the solution vector is equivalent to a pressure vector even if it is the sum of contributions of measured pressure and velocity as shown in Eq. (17). Thus, the presence of multiple corners in L-curve is not the consequence of the combination of pressure and velocity measurements. In addition, Pereira et al. [9] have observed the same phenomenon using only pressure measurements. As the multiple corners problem appears only at eigenfrequencies of the virtual volume, we suspect that the wrong solutions found by CURV-strategy correspond to the presence of solutions of the associate problem that is to say to the eigenmodes of the virtual volume.

\section{Application to a 3D underdetermined problem : the oil pan}

One of the main advantages of iPTF method is its ability to handle complex 3D source surfaces. To illustrate that crucial point, the iPTF method has been applied to identify the velocity, pressure and intensity fields of an oil pan presented in Fig. 13 by measuring the acoustic radiated fields on 


\begin{tabular}{|c|c|}
\hline Number of measurement patches $N_{j}$ & 672 \\
\hline Number of identification patches $N_{k}$ & 930 \\
\hline Maximum frequency of the modal basis & $10 \mathrm{kHz}$ \\
\hline Number of modes & 1516 \\
\hline Number of DoF & 63224 \\
\hline Rank of the matrix $\mathbf{Z}_{i k}$ & 672 \\
\hline
\end{tabular}

TABLE 2: Information on the FE mesh of the virtual volume used to compute impedance matrix $\mathbf{Z}_{i k}$ in the case of the oil pan.

a virtual rectangular box shown in Fig. 14(b). The reference measurements have been acquired on the smallest box surrounding the oil pan, see Fig. 14(a). The reference surface does not correspond to the exact geometry of the source because the measurements would have been to complicated. The identification surface (see Fig. 14(c)) follows the exact geometry of the source thanks to the use of FE mesh of the virtual volume presented in Fig. 14(d). The oil pan was excited by an electro-dynamic shaker in a frequency band from 100 to $5000 \mathrm{~Hz}$. The acoustic pressure and the particle velocity have been measured at the center of each patch of (i) the reference surface and (ii) the virtual surface. An accelerometer glued on the surface of the oil pan has been used as a phase reference.

The impedance matrices have been computed up to $10 \mathrm{kHz}$. The number of modes used to compute the impedance matrix is enough to ensure that the rank of the matrix is driven by the number of patches on the virtual surface and not by the number of modes. The condition number of this matrix is plotted in Fig. 15. As previously remarked, the condition number exhibits peaks at eigenfrequencies of the virtual volume. Some additional information can be found in Tab. 2 .

The mean square pressures are plotted in Fig. 16. As can be seen, results obtained with WCURV and GCV strategies are in good agreement with reference measurement.

Again, as presented in Fig. 17, for some frequencies, the L-curve is not Lshaped. This is the case, for example, at $432 \mathrm{~Hz}$ (Fig. 17(a)). The CURV chooses the $\beta$ value that gives the maximum of curvature but by doing this chooses the wrong solution at this frequency. Again, this solution does not respect the power conservation (power ratio close to 0). Using WCURV-strategy, this solution is weighted by the power ratio and so is highly soften. The first peak which respects the power conservation is chosen by the WCURV- 
strategy.

At $904 \mathrm{~Hz}$, this problem does not appear and the solutions found by CURV and WCURV-strategy are equal.

The velocity fields obtained by both strategies at these two frequencies are presented in Fig. 18. When the L-curve is not L-shaped, the CURV-strategy produces results that are highly dominated by mode shapes of the virtual cavity as visible in Fig. 18(b).

The GCV-strategy produces slightly under-regularized solutions in medium and high frequency as presented in Figs. 16, 17 and 18. The norm of the solution is a little higher than the one obtained by WCURV-strategy. However, this conclusion must be nuanced because of the difference between identification and reference surfaces. In any case, a combinaison between GCV and WCURV strategies may produce satisfactory results in a lot of different applications.

Finally, to demonstrate the accuracy of the fields identified by the iPTF method associated to the WCURV-strategy of regularization, one has plotted the phase of the pressure field in high frequency $(2980 \mathrm{~Hz})$. Very fine details of the highly variating field are reproduced with very good accuracy compared to reference measurements.

\section{Conclusion}

Several acoustic source identification methods exist in the literature. Each of them has its particularities, its advantages and its drawbacks. The inverse Patch Transfer Function method has been developped some years ago to identify the velocity, pressure and intensity field of a $3 \mathrm{D}$ vibrating surface by measuring the radiated acoustic fields (pressure and particle velocity) on an arbitrary virtual surface surrounding the source. The solver used here is based on the computation of an impedance matrix from eigenmodes of the virtual volume (defined by the union of the source surface and the virtual surface of measurements). Neumann's boundary conditions have been chosen even if they don't reflect any reality. As possible in Green's identity, they have been chosen arbitrarily to solve the problem on a basis of orthonormal functions.

However, the matrix to be inverted is ill-conditioned (as in the major part of inverse methods), especially at frequencies corresponding to the eigenfrequencies of the associate problem (eigenmodes of the virtual volume). This results in the appearance of alternative solutions during the Tikhonov re- 
gularization process. At these frequencies, the strategy based on the choice of the regularization parameter corresponding to the maximum of curvature of the L-curve sometimes fails. A wrong solution highly dominated by the eigenmodes of the virtual volume is produced. It has been demonstrated on two real examples (a flat plate and an oil pan) that these solutions don't respect the power conservation. This permits to introduce a weighted curvature function that overcomes the problems observed at frequencies close to eigenfrequencies of the virtual volume. The GCV technique seems to be less affected by the high condition number but can, in some applications, induce a slightly under-regularized solution. A combination of both GCV and WCURV strategies can then be useful.

The iPTF produces then very accurate source fields and gives a lot of information about the source : velocity, wall pressure, active and reactive intensity, acoustic power and radiation efficiency.

\section{Acknowledgements}

This work was performed within the framework of the Labex CeLyA of Université de Lyon, operated by the French National Research Agency (ANR-10-LABX-0060/ ANR-11-IDEX-0007).

\section{References}

[1] M.B.S. Magalhães, R.A. Tenenbaum, Sound sources reconstruction techniques : a review of their evolution and new trends, Acta Acustica united with Acustica, 90 (2004) 199-220.

[2] E.G. Williams, J.D. Maynard, E. Skudrzyk, Sound source reconstructions using microphone array, Journal of the Acoustical Society of America, 68 (1980) 340-344.

[3] J.D. Maynard, E.G. Williams, Y. Lee, Nearfield acoustic holography : I. Theory of generalized holography and the development of NAH, Journal of the Acoustical Society of America, 78 (1985) 1395-1413.

[4] E.G. Williams, H.D. Dardy, Generalized nearfield acoustical holography for cylindrical geometry : Theory and experiment, Journal of the Acoustical Society of America, 81(2) (1987) 389-407. 
[5] F. Jacobsen, G. Moreno-Pescador, E. Fernandez-Grande, J. Hald, Near field acoustic holography with microphones on a rigid sphere, Journal of the Acoustical Society of America, 129(6) (2011) 3461-3464.

[6] G. Chardon, L. Daudet, A. Peillot, F. Ollivier, N. Bertin, R. Gribonval, Near-field acoustic holography using sparse regularization and compressive sampling principles, Journal of the Acoustical Society of America, 132(3) (2012) 1521-1534.

[7] C. Pézerat, Q. Leclère, N. Totaro, M. Pachebat, Identification of vibration excitations from acoustic measurements using near field acoustic holography and the force analysis technique, Journal of Sound and Vibration, 326 (2009) 540-556.

[8] G.H. Koopmann, L. Song, J.B. Fahnline, A method for computing acoustic fields based on the principle of wave superposition, Journal of the Acoustical Society of America, 86(6) (1989) 2433-2438.

[9] A. Pereira, Q. Leclère, J. Antoni, A theoretical and experimental comparison of the equivalent source method and a Bayesian approach to noise source identification, in : Proceedings of Berlin Beamforming Conference, Berlin, 2012.

[10] A. Pereira, Acoustic imaging in enclosed spaces, PhD thesis, INSA-Lyon, 2013.

[11] M.R. Bai, Application of BEM (boundary element method)-based acoustic holography to radiation analysis of sources with arbitrarily shaped geometries, Journal of the Acoustical Society of America, 92(1) (1992) 533-549.

[12] B.-K. Kim, J. Ih, On the reconstruction of the vibro-acoustic field over the surface enclosing an interior space using the boundary element method, Journal of the Acoustical Society of America, 100(5) (1996) 30033016.

[13] C. Langrenne, M. Melon, A. Garcia, Boundary element method for the acoustic characterization of a machine in bounded noisy environment, Journal of the Acoustical Society of America, 121(5) (2007) 2750-2757. 
[14] M. Aucejo, N. Totaro, J.-L. Guyader, Identification of source velocities on 3D structures in non-anechoic environments : Theoretical background and experimental validation of the inverse patch transfer functions method, Journal of Sound and Vibration, 329 (2010) 3691-3708.

[15] T. Kletschkowski, M. Weber, D. Sachau, Identification of noise source in an aircraft fuselage using an inverse method based on a finite element model, Acta Acustica united with Acustica, 97 (2011) 974-983.

[16] D. Vigoureux, Déconfinement de sources acoustiques par utilisation d'une méthode holographique à double information, $\mathrm{PhD}$ thesis, 2012.

[17] C. Langrenne, A. Garcia, Data completion method for the characterization of sound sources, Journal of the Acoustical Society of America, 130(4) (2011) 2016-2023.

[18] E. Fernandez-Grande, F. Jacobsen, Q. Leclère, Sound field separation with sound pressure and particle velocity measurements, Journal of the Acoustical Society of America, 132(6) (2012) 3818-3825.

[19] Y. Braikia, M. Melon, C. Langrenne, E. Bavu, A. Garcia, Evaluation of a separation method for source identification in small spaces, Journal of the Acoustical Society of America, 134(1) (2013) 323-331.

[20] M. Aucejo, N. Totaro, Guyader J.L., Identification of source velocities in presence of external correlated sources with the inverse Patch Transfer Functions (iPTF) method, in : Proceedings of NOVEM 2009, Oxford, UK, 2009.

[21] M. Aucejo, N. Totaro, J.L. Guyader, Identification of source velocities with Inverse Patch Transfer Functions method, in : proceedings of ACOUSTICS' 08, Paris, France, 2008.

[22] D. Vigoureux, N. Totaro, J.L. Guyader, Mesure de vitesse vibratoire par la méthode iPTF : simulation et expérimentation, in : Proceedings of 10eme Congres Francais d'Acoustique, Lyon, France, 2010.

[23] N. Totaro, C. Sandier, J.L. Guyader, Identify velocity of a complex source with iPTF method, in : proceedings of ICSV15, Daejon, Korea, 2008 . 
[24] N. Totaro, C. Sandier, Q. Leclère, Source identification on a reduction gearbox using acoustical measurements in a non-anechoic environment, in : Proceedings of ISCV19, Vilnius, Lithuania, 2012.

[25] P.C. Hansen, Rank-deficient and discrete ill-posed problems (SIAM, Philadelphia, PA) (1998).

[26] E.G. Williams, Regularization methods for near-field acoustical holography, Journal of the Acoustical Society of America, 110(4) (2001) 19761988 .

[27] F. Jacobsen, V. Jaud, A note on the calibration of the pressure-velocity sound intensity probes, Journal of the Acoustical Society of America, 120(2) (2006) 830-837.

[28] J. Antoni, A Bayesian approach to sound source reconstruction : optimal basis, regularization, and focusing, Journal of the Acoustical Society of America, 131(4) (2012) 2873-2890.

[29] P.K. Venkatesh, On Tikhonov regularisation, Physica A 284 (2000) 448460.

[30] P. Simard, J. Antoni, Acoustic source identification : experimenting the $l_{1}$ minimization approach, Applied Acoustics, 74 (2013) 974-986.

[31] P.-A. Gauthier, A. Gérard, C. Camier, A. Berry, Acoustical inverse problems regularization : Direct definition of filter factors using signal-tonoise Ratio, Journal of Sound and Vibration, 333 (2014) 761-773.

[32] Q. Leclère, Acoustic imaging using under-determined inverse approaches : Frequency limitations and optimal regularization, Journal of Sound and Vibration, 321 (2009) 605-619.

[33] Y. Kim, P.A. Nelson, Spatial resolution limits for the reconstruction of acoustic source strength by inverse methods, Journal of Sound and Vibration, 265 (2003) 583-608.

[34] Y. Kim, P.A. Nelson, Optimal regularisation for acoustic source reconstruction by inverse methods, Journal of Sound and Vibration, 275 (2004) 463-487. 
[35] H.G. Choi, A.N. Thite, D.J. Thompson, Comparison of methods for parameter selection in Tikhonov regularization with application to inverse force determination, Journal of Sound and Vibration, 304 (2007) 894-917.

[36] M.J. Crocker, A. J. Price, Sound transmission using Statistical Energy Analysis, Journal of Sound and Vibration 9 (1969) 469-486. 


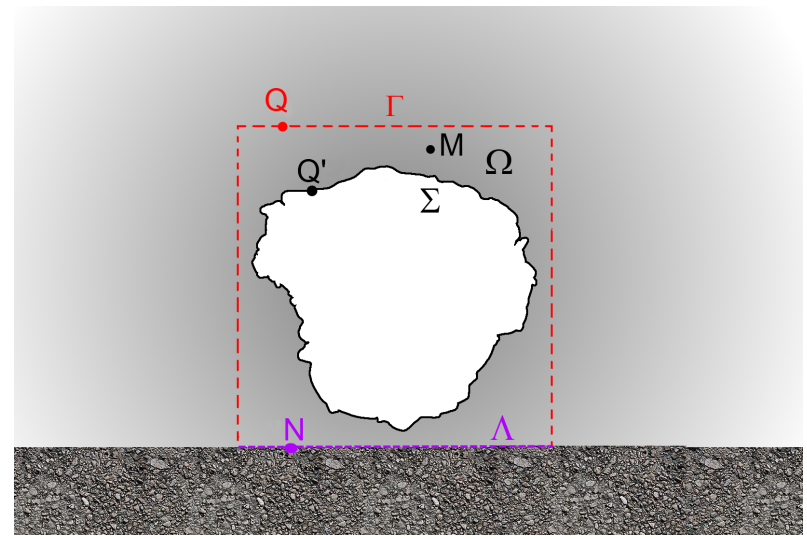

Figure 1: Definition of the finite acoustic volume $\Omega$ (virtual volume) and the boundary surfaces $\Sigma$ (surface of the vibrating object), $\Gamma$ (virtual surface surrounding the object) and $\Lambda$ (physically rigid surface). 


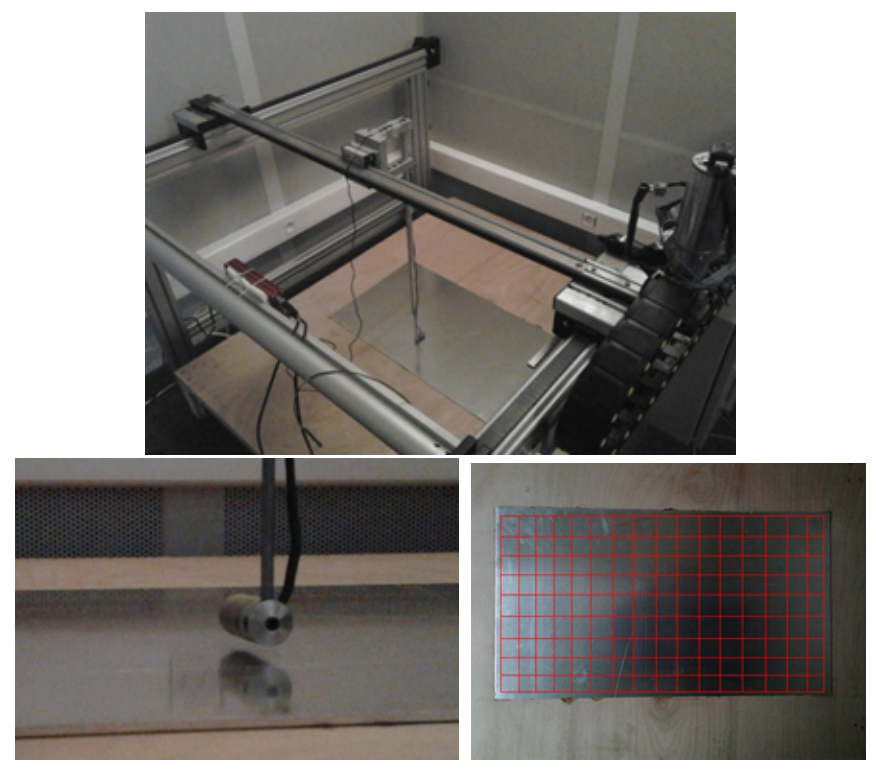

Figure 2: Photographies of the baffled plate under study. The measures of the pressures and the acoustic velocities have been done using a pU probe. The plate is excited by a shaker fed with white noise. 


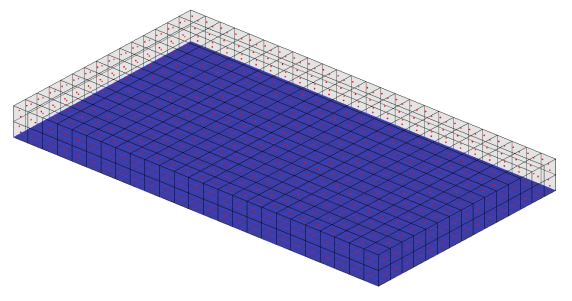

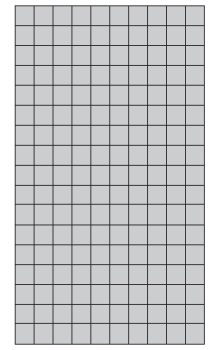

(a)

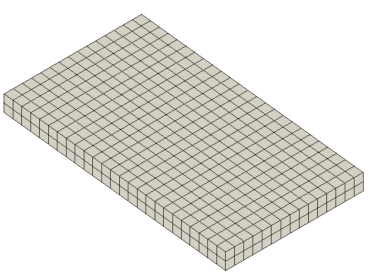

(b)

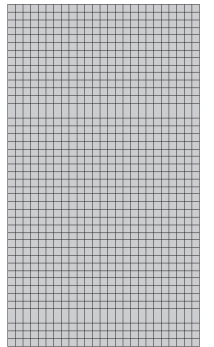

(c)

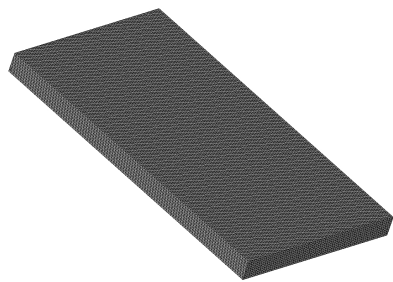

(d)

Figure 3: Meshes of the patches used for (a) reference measurements; (b) radiated acoustic fields measurements; (c) identification. (d) FE mesh of the virtual volume. 


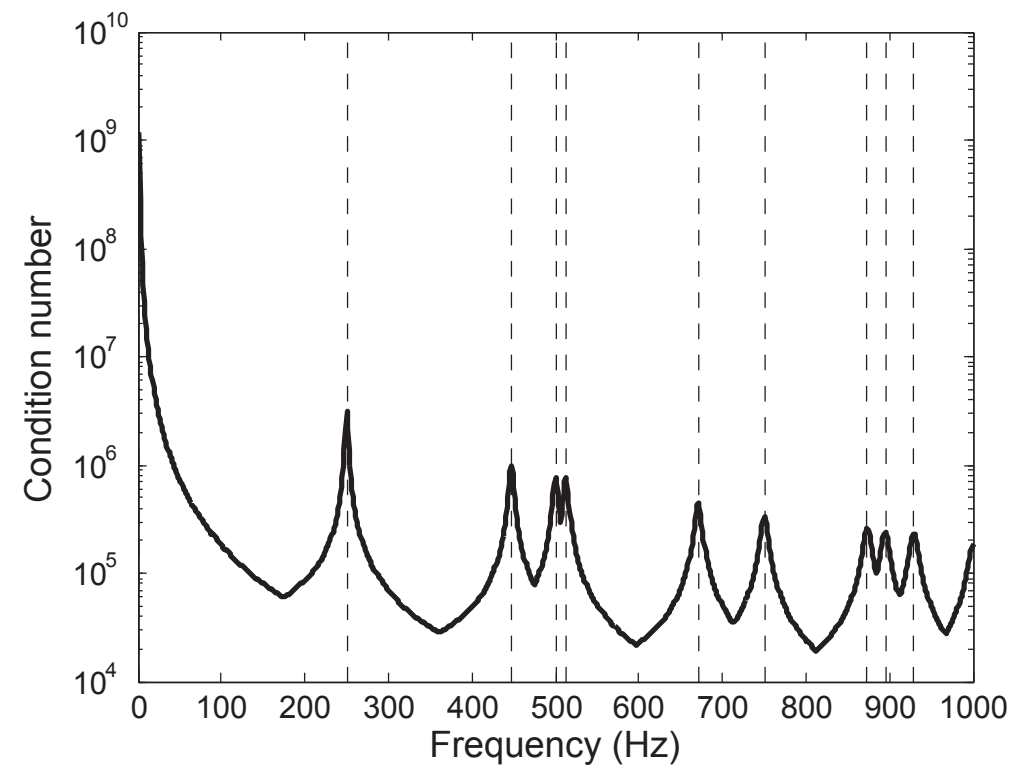

Figure 4: Condition number of the matrix to be inverted. The dashed lines represent the positions of the eigen-frequencies of the virtual cavity. 


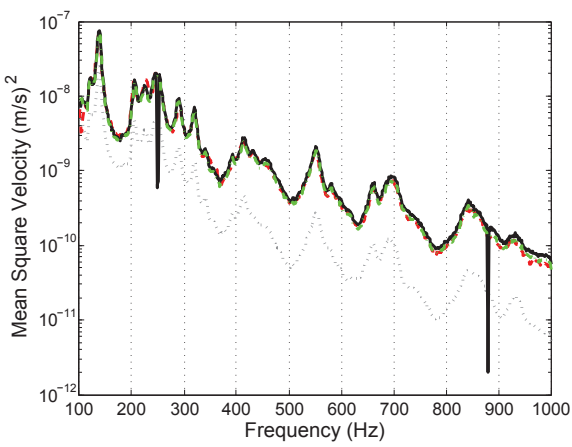

(a)

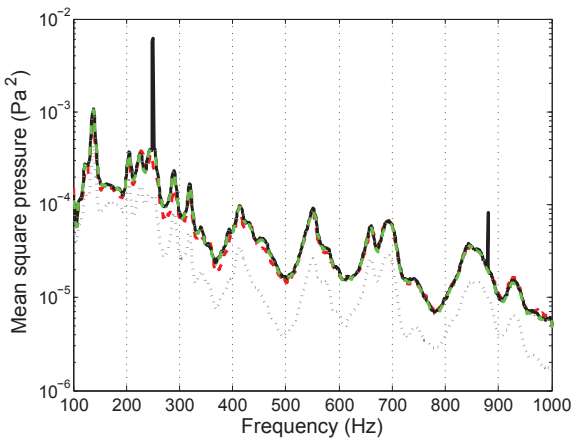

(c)

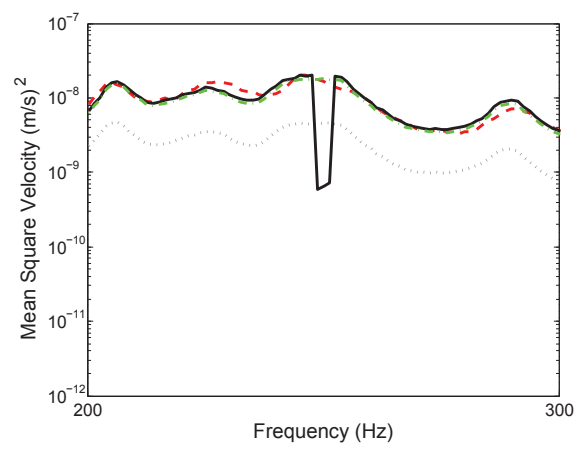

(b)

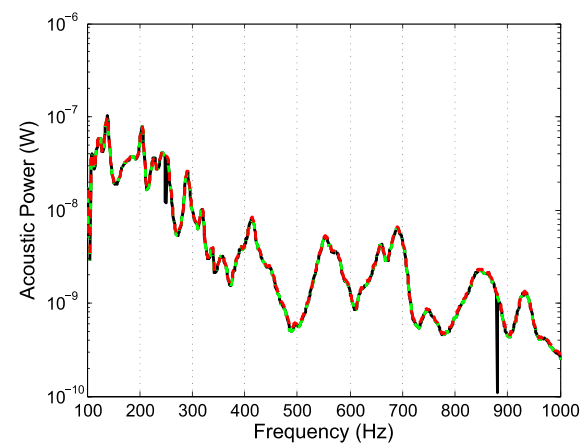

(d)

Figure 5: (a) Mean square velocity; (b) Mean square velocity in the frequency band $[200 \ldots 300] \mathrm{Hz}$; (c) Mean square pressure and (d) Acoustic power. Red dashed line : reference measurement; dotted gray line : radiated acoustic field measurement; black solid line : identification with CURV-strategy, green dashed-dotted line : identification with GCVstrategy. 


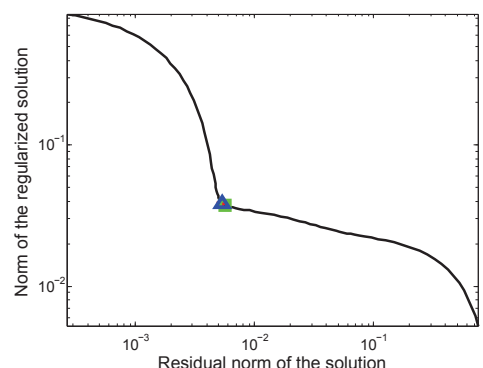

(a) L-curve $\mathrm{f}=137.5 \mathrm{~Hz}$

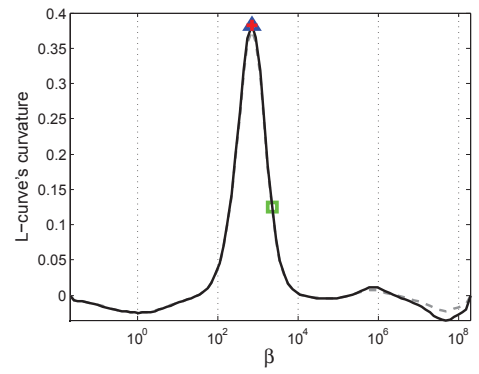

(c) Curvature $\mathrm{f}=137.5 \mathrm{~Hz}$

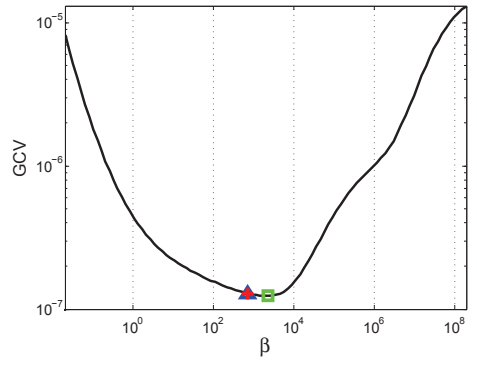

(e) $\mathrm{GCV} \mathrm{f}=137.5 \mathrm{~Hz}$

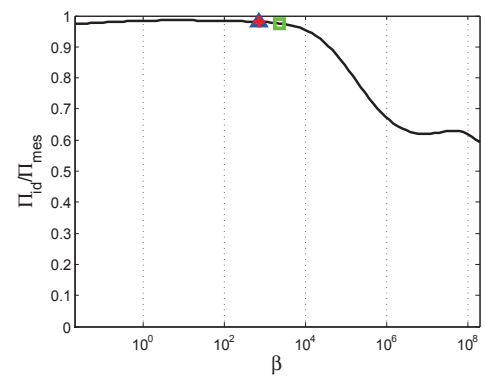

(g) Power ratio $\mathrm{f}=137.5 \mathrm{~Hz}$

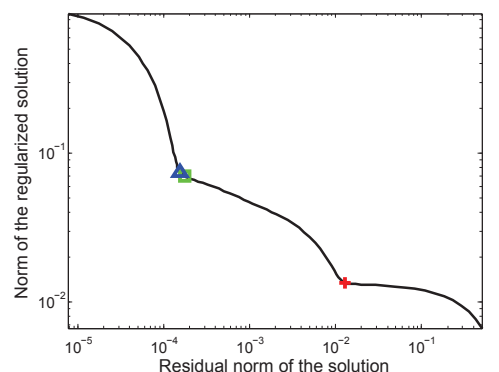

(b) L-curve $\mathrm{f}=250 \mathrm{~Hz}$

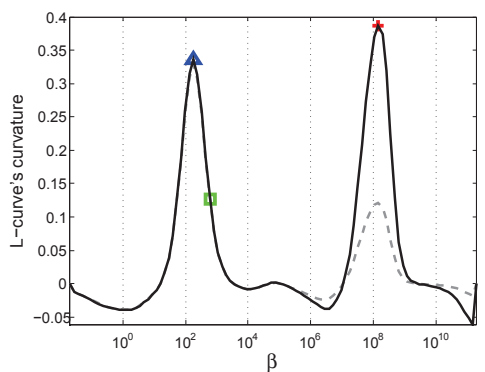

(d) Curvature $\mathrm{f}=250 \mathrm{~Hz}$

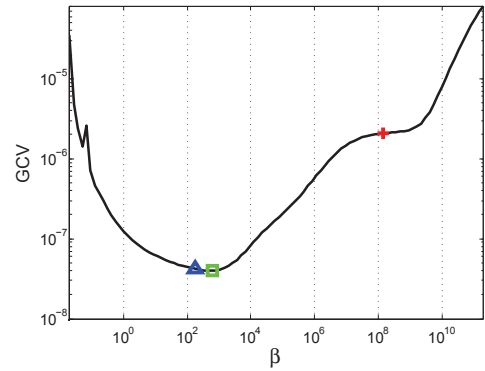

(f) $\mathrm{GCV} \mathrm{f}=250 \mathrm{~Hz}$

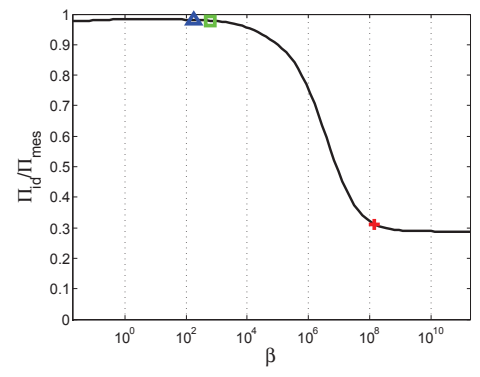

(h) Power ratio $\mathrm{f}=250 \mathrm{~Hz}$

Figure 6: (a)-(b) L-curve, (c)-(d) curvature of the L-curve, (e)-(f) GCV and (g)-(h) power ratio as a function of regularization parameter at frequencies $137.5 \mathrm{~Hz}$ and $250 \mathrm{~Hz}$. Crosses : Curv-strategy; Triangles : wCurV-strategy; Squares : GCV-strategy. The dashed lines in (c) and (d) represent the weighted curvature function defined by Eq. (23) 


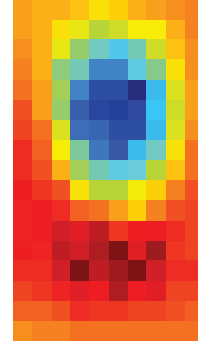

(a) Ref : $V$

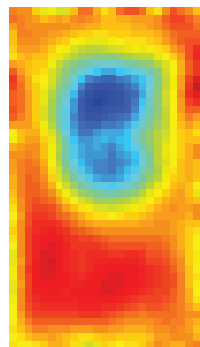

(e) GCV : $V$

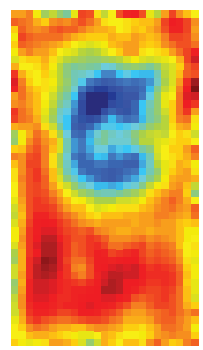

(i) Curv : V

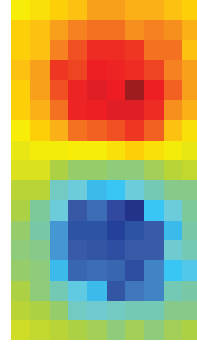

(b) Ref : $P$

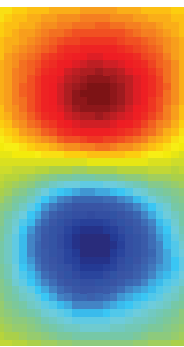

(f) $\mathrm{GCV}: P$

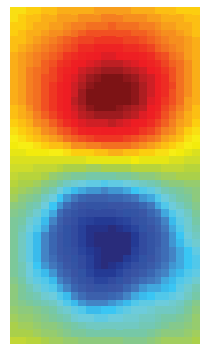

(j) Curv : $P$

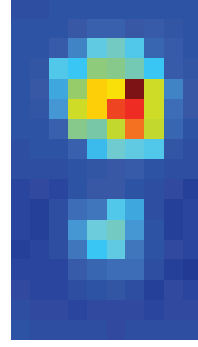

(c) Ref : $I_{a c}$

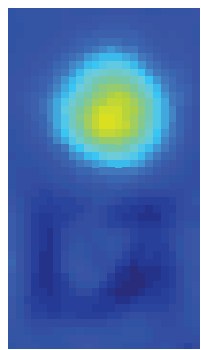

(g) GCV : $I_{a c}$

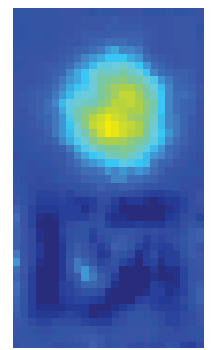

(k) Curv : $I_{a c}$

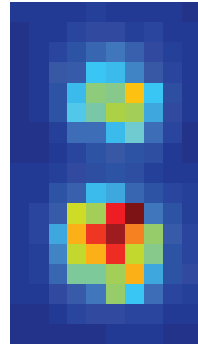

(d) Ref : $I_{r e}$

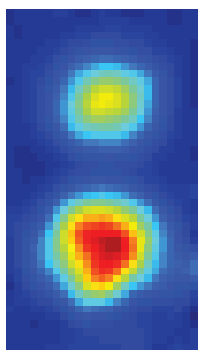

(h) GCV $: I_{r e}$

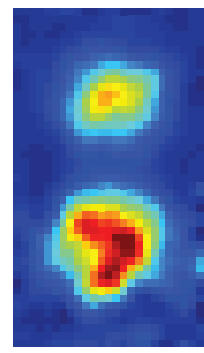

(1) Curv : $I_{r e}$

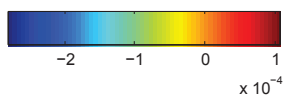

$\begin{array}{ccccc}-0.06 & -0.04 & -0.02 & 0 & 0.02\end{array}$

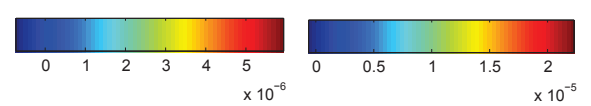

FiguRE 7: Comparison between reference measurements ((a)-(d)) and identification results obtained with GCV ((e)-(h)) and CURv ((i)-(l)) strategies in terms of velocity $\left(V, \mathrm{~m} . \mathrm{s}^{-1}\right)$, pressure $(P, \mathrm{~Pa})$, active intensity $\left(I_{a c}, \mathrm{~W} \cdot \mathrm{m}^{-2}\right)$ and reactive intensity $\left(I_{r e}, \mathrm{~W} \cdot \mathrm{m}^{-2}\right)$ fields at $137.5 \mathrm{~Hz}$. 


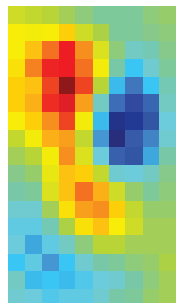

(a) Ref : $V$

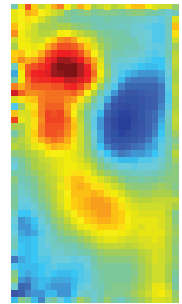

(e) GCV : $V$

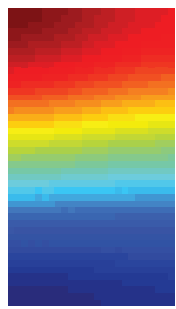

(i) Curv : $V$

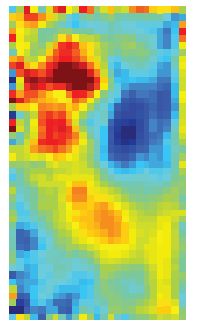

(m) wCurv : $V$

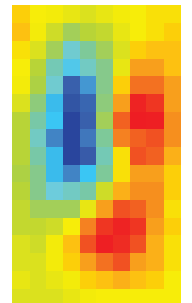

(b) Ref : $P$

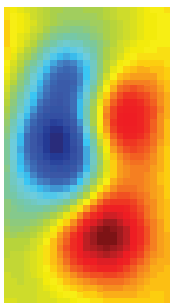

(f) GCV : $P$

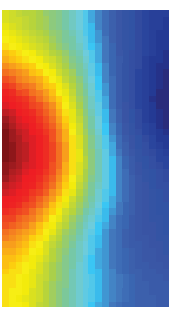

(j) Curv : $P$

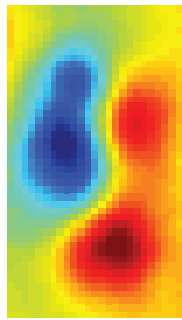

(n) wCurv : $P$

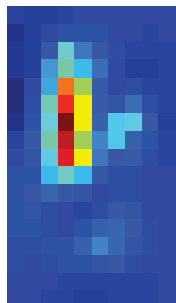

(c) Ref : $I_{a c}$

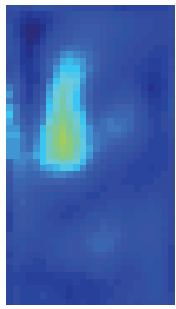

(g) GCV : $I_{a c}$

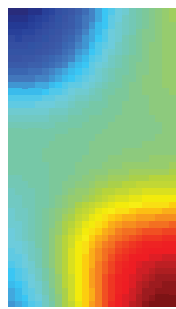

(k) Curv : $I_{a c}$
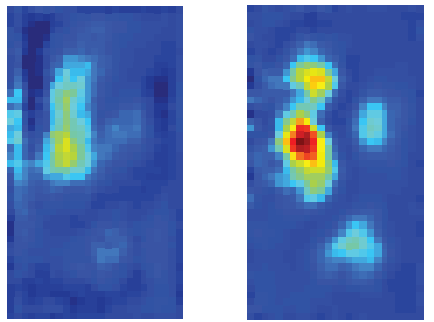

(o) wCurv : $I_{a c}$ (p) wCurv : $I_{r e}$
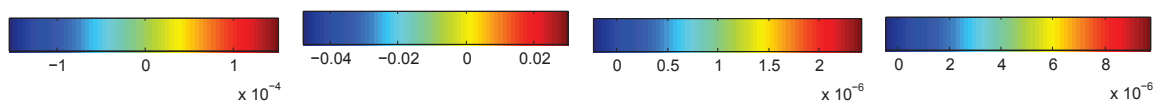

FiguRE 8: Comparison between reference measurements ((a)-(d)) and identification results obtained with GCV ((e)-(h)), CURV ((i)-(l)) and wCuRV ((m)-(p)) strategies in terms of velocity $\left(V, \mathrm{~m} \cdot \mathrm{s}^{-1}\right)$, pressure $(P, \mathrm{~Pa})$, active intensity $\left(I_{a c}, \mathrm{~W} \cdot \mathrm{m}^{-2}\right)$ and reactive intensity $\left(I_{r e}, \mathrm{~W} \cdot \mathrm{m}^{-2}\right)$ fields at $250 \mathrm{~Hz}$. 


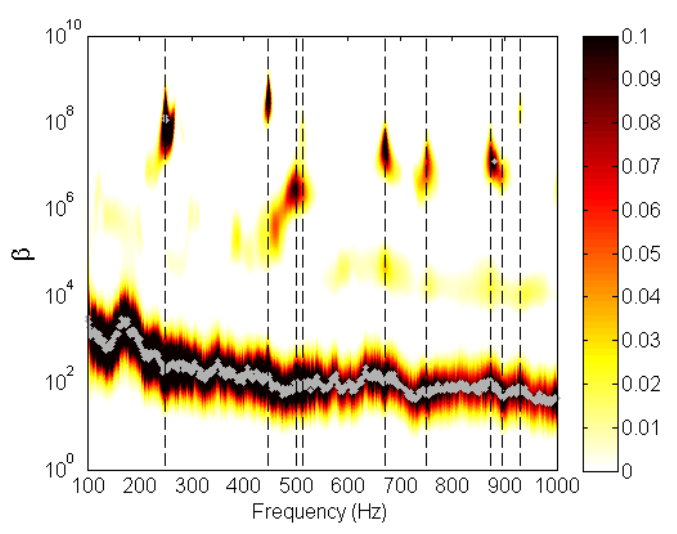

(a)

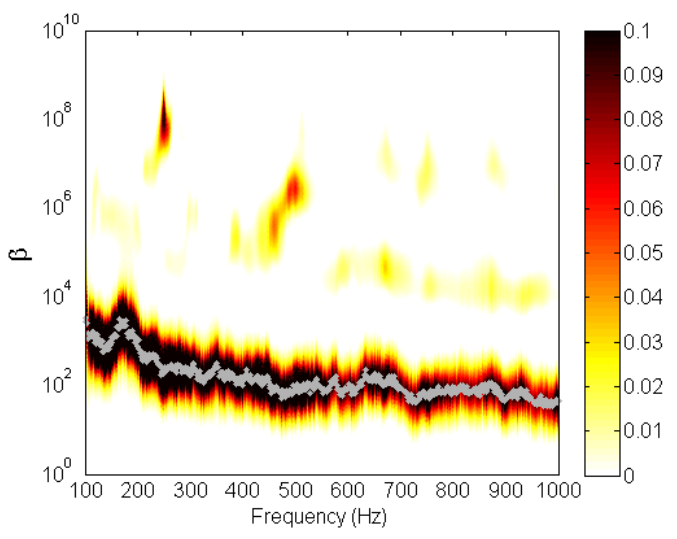

(b)

FiguRE 9: (a) curvature of L-curve and (b) weighted curvature of L-curve as a function of frequency and regularization parameter $\beta$.

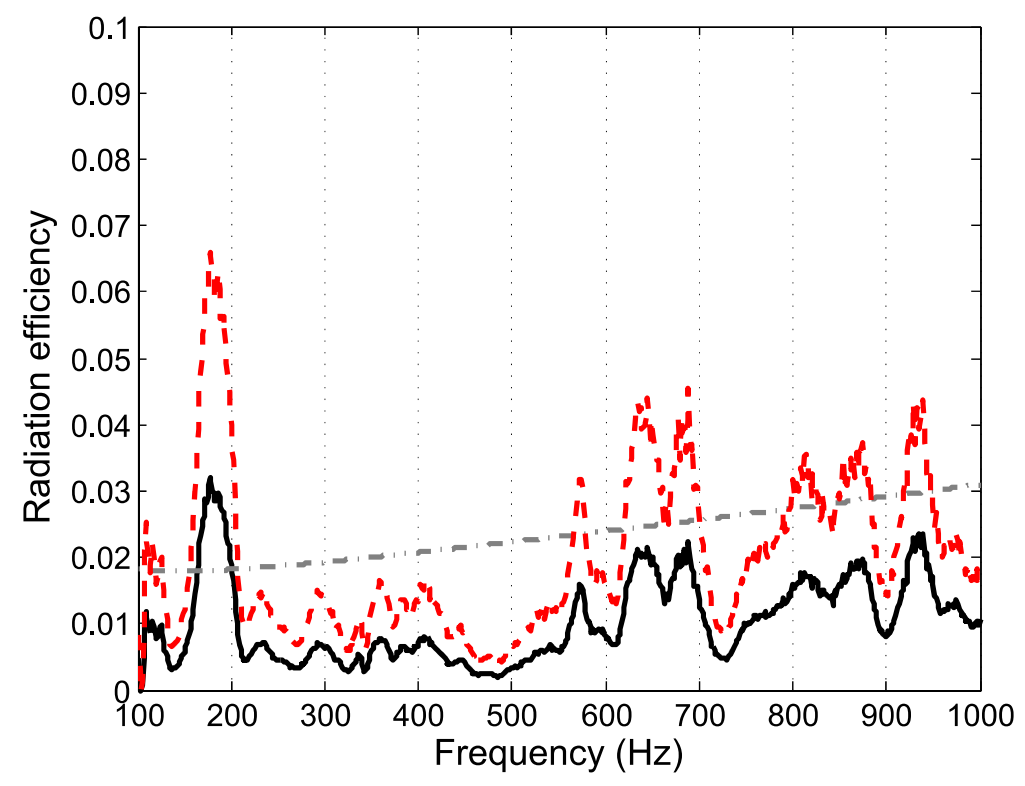

Figure 10: Radiation efficiency of the plate. Solid black line : identification with wCuRVstrategy ; dotted red line : identification with GCV-strategy; dashed-dotted line : Crocker and Price's model [36] 


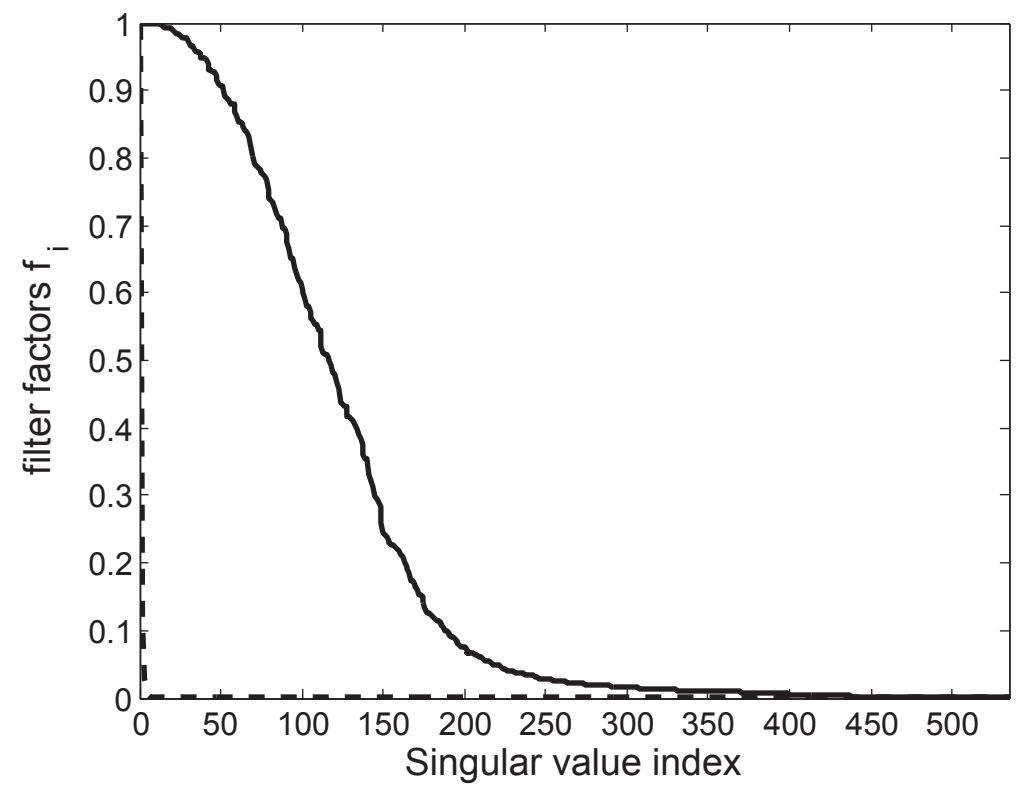

Figure 11: Filter factor $f_{m}$ for $\beta=179.5$ (first peak of curvature, solid line) and for $\beta=1.416 \times 10^{8}$ (second peak of curvature, dashed line) at $250 \mathrm{~Hz}$ as a function of singular value index. 


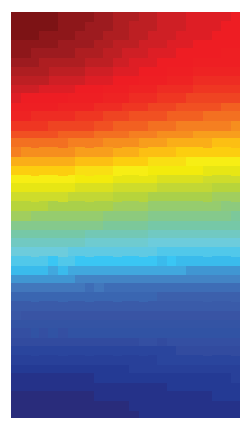

(a)

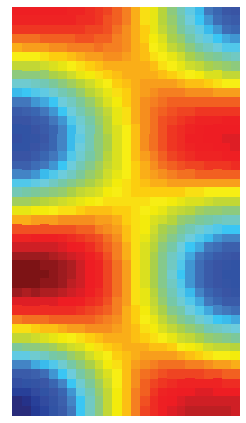

(c)

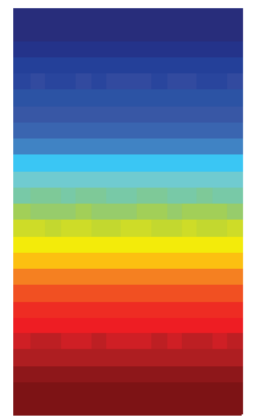

(b)

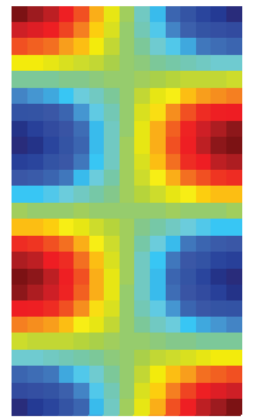

(d)

Figure 12: Comparison between the identified velocity field using CuRV-strategy and projection of pressure mode shapes of the virtual cavity on the identification surface. (a) identified velocity field at $250 \mathrm{~Hz}$; (b) projection of the second mode (eigenfrequency $=250 \mathrm{~Hz}$ ) ; (c) identified velocity field at $880 \mathrm{~Hz}$; (d) projection of the eighth mode (eigenfrequency $=873.5 \mathrm{~Hz})$. 


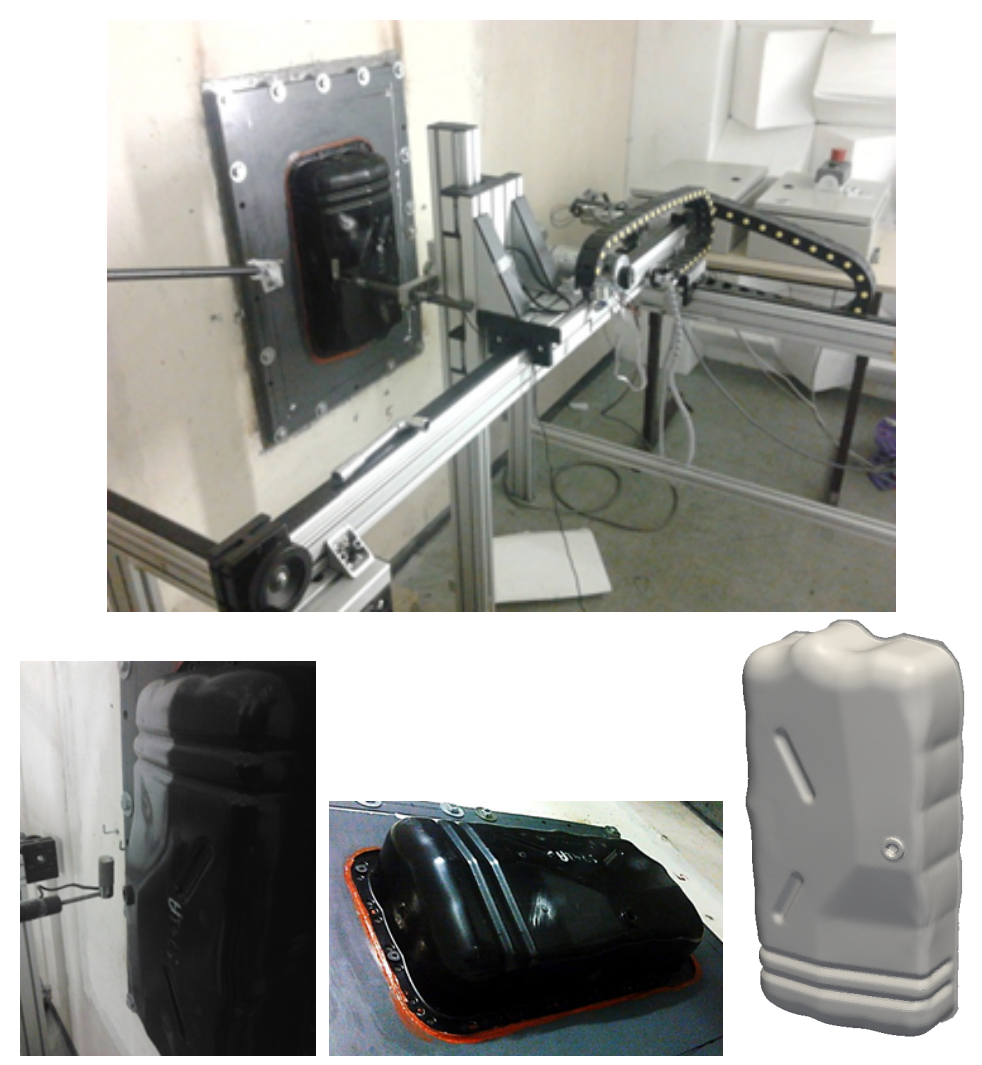

Figure 13: Photographies of the baffled oil pan under study. The measures of the pressures and the acoustic velocities have been done using a pU probe. The oil pan is excited by a shaker fed with white noise. 


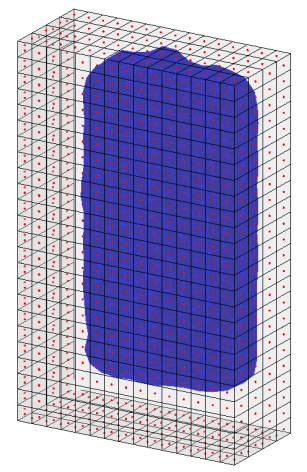

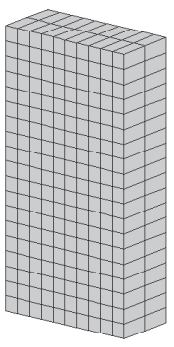

(a)

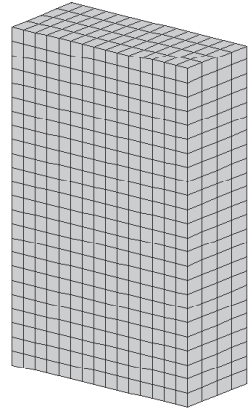

(b)

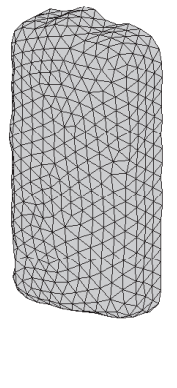

(c)

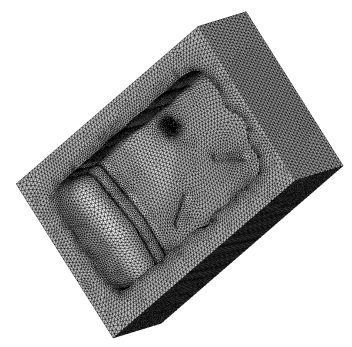

(d)

FiguRE 14: Meshes of the patches used for (a) reference measurements; (b) radiated acoustic fields measurements; (c) identification. (d) FE mesh of the virtual volume. 


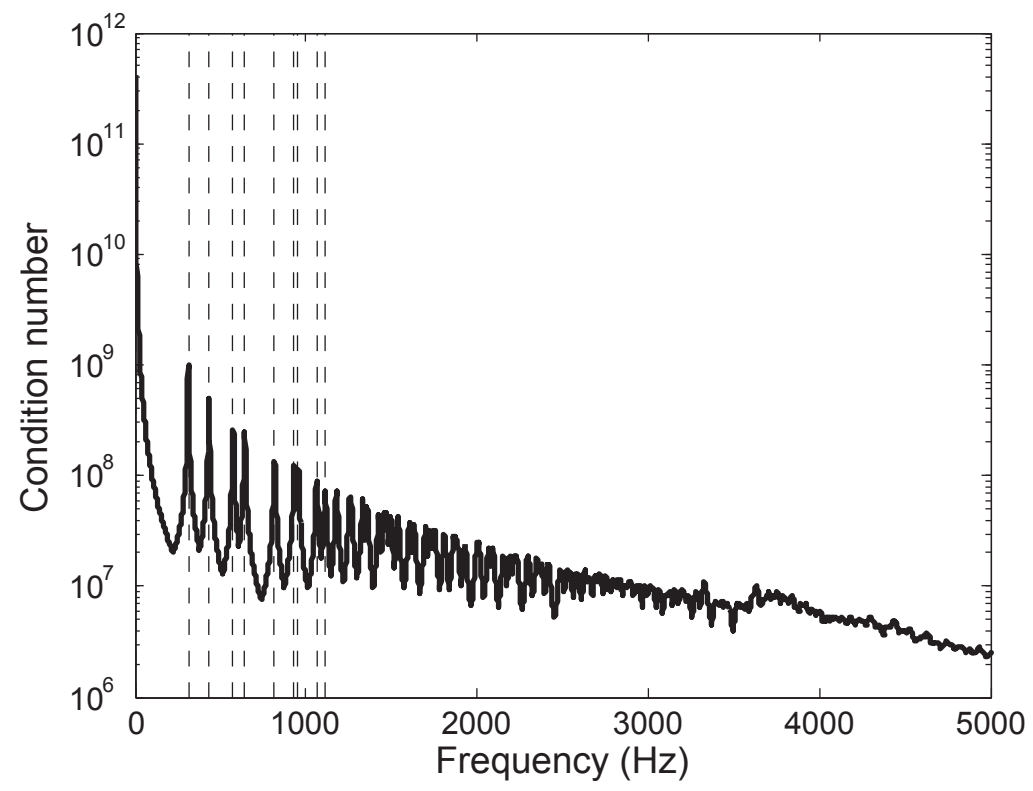

Figure 15: Condition number of the matrix to be inverted. The dashed lines represent the positions of the ten first eigen-frequencies of the virtual cavity.

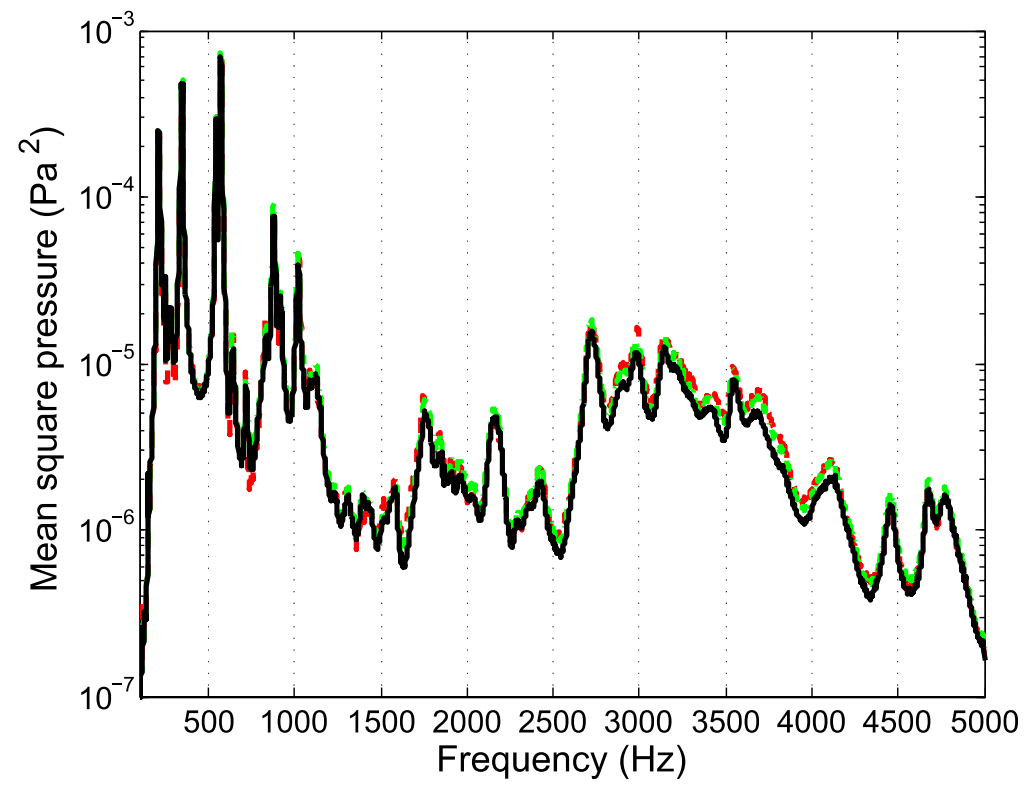

FiguRE 16: Mean square pressure. Red dashed line : reference measurements, black solid line : identification with CuRV-strategy, green dashed-dotted line : identification with GCV-strategy. 


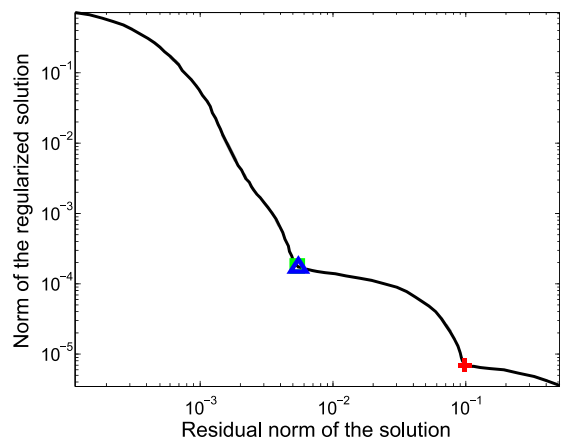

(a) L-curve $432 \mathrm{~Hz}$

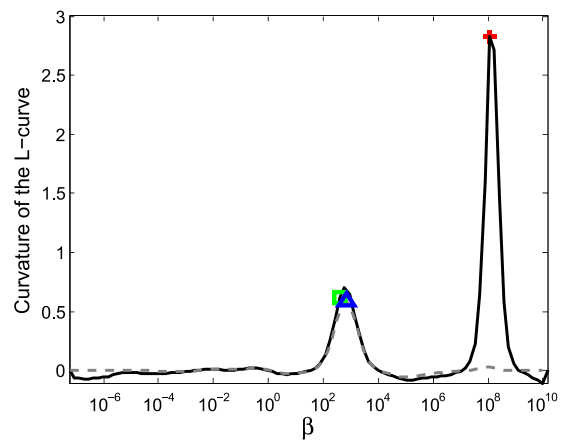

(c) Curvature $432 \mathrm{~Hz}$

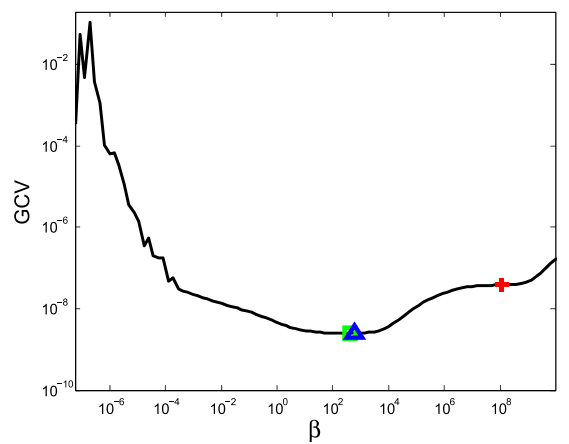

(e) $\mathrm{GCV} 432 \mathrm{~Hz}$

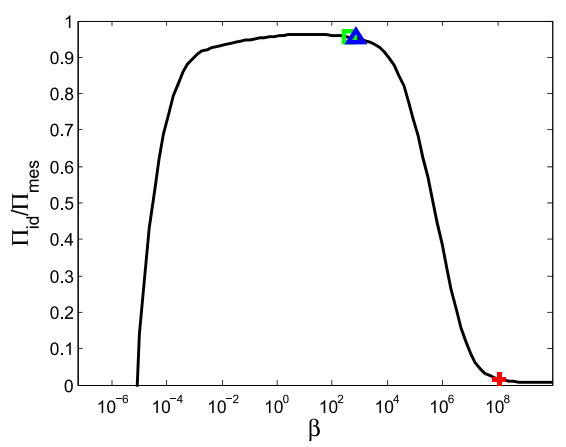

(g) Power ratio $432 \mathrm{~Hz}$

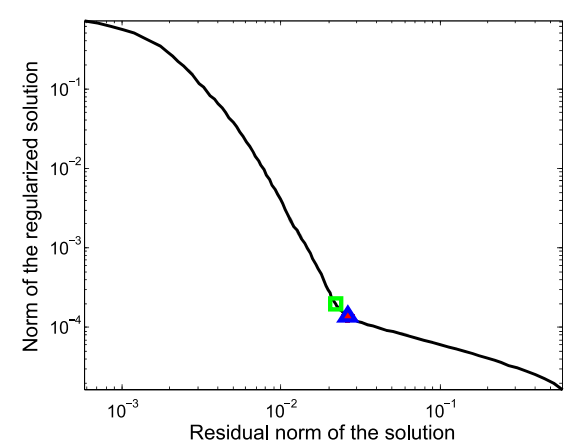

(b) L-curve $904 \mathrm{~Hz}$

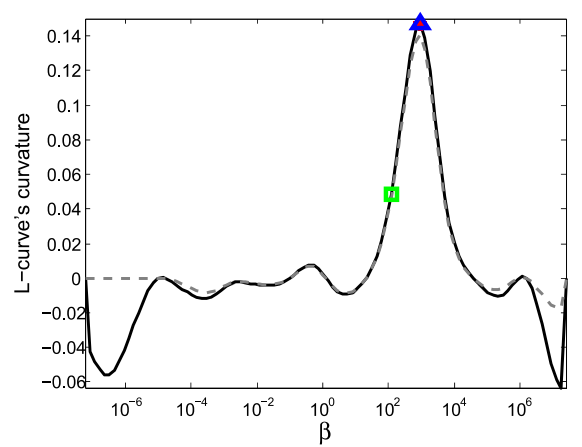

(d) Curvature $904 \mathrm{~Hz}$

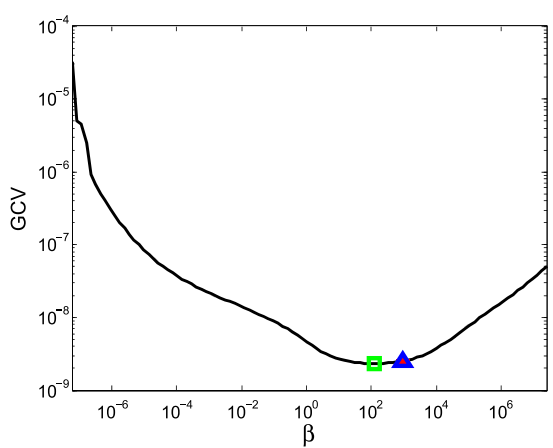

(f) $\mathrm{GCV} 904 \mathrm{~Hz}$

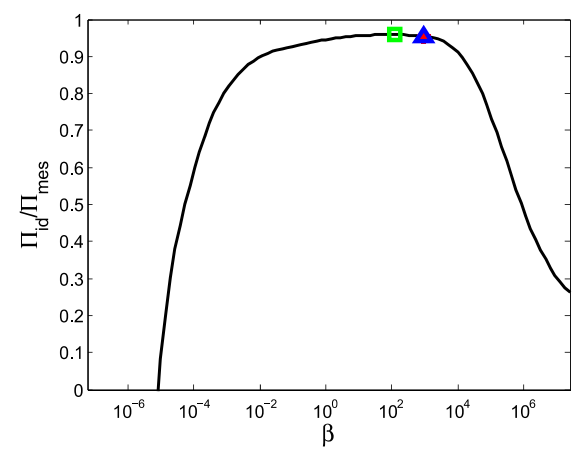

(h) Power ratio $904 \mathrm{~Hz}$

Figure 17: (a)-(b) L-curve, (c)-(d) curvature of the L-curve, (e)-(f) GCV and (g)-(h) power ratio as a function of regularization parameter at frequencies $432 \mathrm{~Hz}$ and $904 \mathrm{~Hz}$. Crosses : Curv-strategy; Triangles : wCuRV-strategy; Squares : GCV-strategy. 


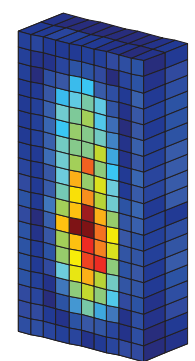

(a) Ref

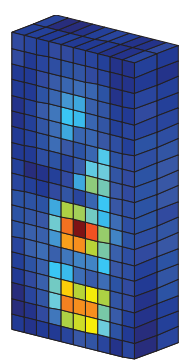

(e) Ref

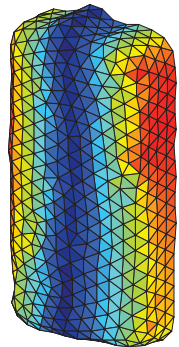

(b) CuRv

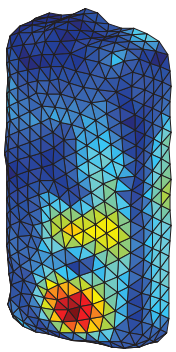

(f) Curv

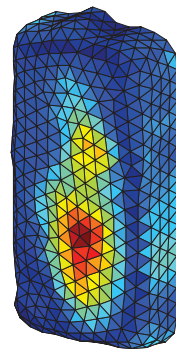

(c) WCURv

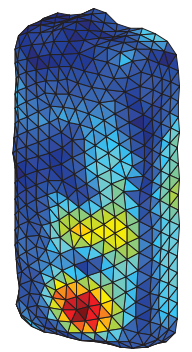

(g) WCURv

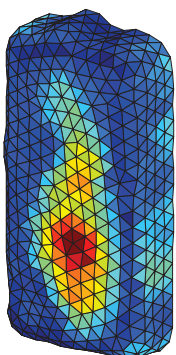

(d) GCV

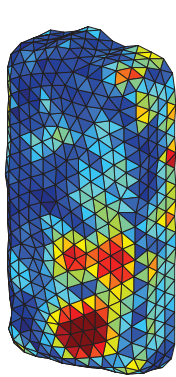

(h) GCV
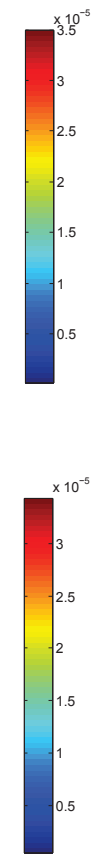

FIgURE 18: Amplitude of the source velocity field $\left(\mathrm{m} . \mathrm{s}^{-1}\right)$. Comparison between reference measurements and identification results obtained with CuRv, WCURV and GCV strategies at $432 \mathrm{~Hz}(\mathrm{a})-(\mathrm{d})$ and at $904 \mathrm{~Hz}(\mathrm{e})-(\mathrm{h})$. Scale of subfigure (b) is not the same, it is $0.0109 \times$ $10^{-6}$ to $0.7860 \times 10^{-6}$.
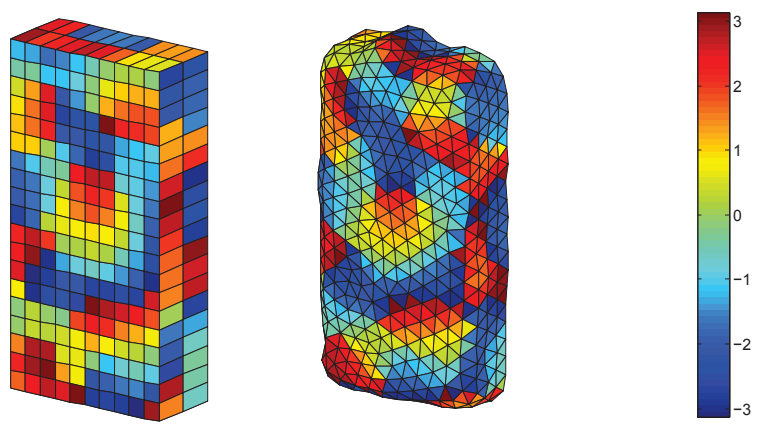

Figure 19: Phase (rad) of the pressure field at 2980Hz. (a) reference measurements; (b) WCURV strategy. 